\title{
Anomia y Alienación en Estudiantes Secundarios de Santiago de Chile: Resultados Iniciales de un Estudio Comparativo 1989-2007
}

\section{Anomie and Alienation in High School Students in Santiago of Chile: Initial Results of a Comparative Research Study 1989-2007}

\author{
Roberto Aceituno, Rodrigo Asún y Soledad Ruiz \\ Universidad de Chile \\ Alejandro Reinoso \\ Pontificia Universidad Católica de Chile \\ Juan Ignacio Venegas y Francisca Corbalán \\ Universidad de Chile
}

\begin{abstract}
Se presentan los avances de un estudio sobre percepción de integración social y normativa en estudiantes de cuarto año de educación media de Santiago $(N=343)$. Se analizan los resultados de un cuestionario sobre anomia y alienación psicosocial, validado en un estudio análogo realizado en 1989. La replicación en 2007 contribuye a la caracterización de la experiencia de integración social y normativa en los jóvenes de hoy, así como al análisis de sus transformaciones casi 20 años después. Los resultados muestran que no existe una clara diferencia en los niveles de anomia y alienación, respecto a los de 1989 .
\end{abstract}

Palabras clave: juventud, anomia, alienación, integración social.

\begin{abstract}
In a sample of Chilean students in their last year of high school $(N=343)$ anomie and psychosocial alienation were measured with a questionnaire validated in an analogous study done in 1989 . This study compared the results of 1989 with those of 2007. This comparison contributes to the psychosocial characterization of social and normative integration experiences of Chilean youth today, and to the analysis of their transformations approximately 20 years later. The results show that there is not a clear difference in the social and normative integration levels in the studied youth, with respect to those of 1989 .
\end{abstract}

Keywords: youth, anomie, alienation, social integration.

\footnotetext{
Roberto Aceituno, Departamento de Psicología, Facultad de Ciencias Sociales, Universidad de Chile, Santiago, Chile. Rodrigo Asún, Departamento de Sociología, Facultad de Ciencias Sociales, Universidad de Chile, Santiago, Chile. Soledad Ruiz, Departamento de Psicología, Facultad de Ciencias Sociales, Universidad de Chile, Santiago, Chile. Alejandro Reinoso, Escuela de Psicología, Pontificia Universidad Católica de Chile, Santiago, Chile.

Juan Ignacio Venegas, Tesista del Departamento de Sociología, Facultad de Ciencias Sociales, Universidad de Chile, Santiago, Chile.

Francisca Corbalán, Asistente de investigación, Departamento de Psicología, Facultad de Ciencias Sociales, Universidad de Chile, Santiago, Chile.

La correspondencia relativa a este artículo debe ser dirigida a Roberto Aceituno, Departamento de Psicología, Facultad de Ciencias Sociales, Universidad de Chile, Ignacio Carrera Pinto 1045, Santiago, Chile. E-mail: raceitun@uchile.cl El artículo reporta los resultados de un proyecto de investigación en Ciencias Sociales, Humanidades y Educación 2006: Integración normativa en jóvenes. Un estudio comparativo 1989-2006, SOC 06/25-2, Vicerrectoría de Investigación y Desarrollo, Universidad de Chile.
} 


\section{Presentación}

El estudio de la subjetividad juvenil chilena requiere considerar la percepción que los jóvenes tienen respecto a su integración normativa y social, así como los eventuales cambios que esta experimenta a la luz de las transformaciones producidas durante los últimos 20 años en Chile. Se entiende por integración normativa y social la experiencia subjetiva de los jóvenes respecto al ordenamiento social percibido, sea en función de su condición etaria (jóvenes o adolescentes), su identidad-diferencia generacional (respecto a la percepción sobre la experiencia psicosocial de integración en la generación anterior) o en función de su condición socioeconómica, entre otros aspectos. Indagar en esta dimensión subjetiva de la integración se muestra relevante si se atiende al propósito de construir una sociedad en la que los ciudadanos no solo estén materialmente integrados sino que, también, tengan sentimientos de pertenencia e inclusión social.

Los conceptos de anomia y alienación han servido como referentes teóricos para caracterizar dichos procesos de integración (o exclusión) social, bajo el supuesto que a través de tales fenómenos se expresan, por una parte, las condiciones que la sociedad ofrece a los individuos para su desarrollo y, por otra, la percepción que estos tienen respecto de la organización (o desorganización) social y normativa que dichas condiciones les plantean. El fenómeno de anomia da cuenta de la relación de los individuos con el mundo social del que forman parte, específicamente respecto a la percepción sobre el "orden" provisto por las normas que organizan su experiencia como individuos. Por otra parte, la alienación pone el acento en la percepción de integración (o desintegración) social respecto a dicho mundo, a través de los vínculos experimentados con otros, de la percepción acerca de las oportunidades y restricciones que ofrece la sociedad para su desarrollo y de la posibilidad de incidir en las condiciones de vida que les son propias.

Teniendo como base teórico-metodológica estas categorías psicosociales, las preguntas que orientan este estudio se dirigen, por una parte, a caracterizar la percepción de integración social y normativa en una muestra de estudiantes de último año de enseñanza media en Santiago de Chile, medida a través de escalas de anomia y alienación que fueron validadas y utilizadas en el año 1989 en un estudio análogo (Aceituno, 1989) y, por otra parte, a comparar los resultados de dicho estudio con los obtenidos en una aplicación realizada en 2007.

La investigación aquí reportada describe la pertinencia de las categorías utilizadas para dar cuenta de esta realidad, a la vez subjetiva y social. Desde una perspectiva metodológica, el estudio realizado permite discutir la construcción de las escalas utilizadas, así como su coherencia teórica a la luz de los resultados obtenidos.

\section{Antecedentes}

\section{Breve Reseña Histórica acerca de la Investigación sobre Juventud en Chile}

La investigación en juventud en Chile ha cambiado de eje focal en los últimos 50 años, siguiendo la evolución del contexto social e intelectual en que las distintas generaciones han vivido este período etario.

En primer lugar, existe en nuestro país una larga tradición científica empirista asociada a los temas de salud pública, que profundiza en la noción de adolescencia como etapa crucial en el itinerario de vida en la cual la toma de decisiones y sus eventuales riesgos son fundamentales en el proceso de integración e inclusión social. Esta mirada se centra en un enfoque clínico epidemiológico, que deriva hacia los años 60 en una formulación crítica de las desiguales condiciones en que los distintos grupos sociales de jóvenes tienen que vivir este período. Los conceptos de riesgo social y el estudio del consumo juvenil de drogas y alcohol arrancan desde esta perspectiva, aún en vigencia en algunos círculos de investigadores.

Durante los años 60, el aumento de la escolarización secundaria y la emergencia política de la juventud universitaria tienen como consecuencia la deriva de la investigación en juventud hacia su estudio en cuanto actor social. Esta línea de investigación le atribuye a los jóvenes 
actitudes casi endógenas de rebeldía e inconformismo con la situación social del momento (Agurto, Canales \& De la Maza, 1985). Si bien en esos tiempos algunas investigaciones emblemáticas ponían en duda la extensión del sentimiento a favor del cambio social en los jóvenes chilenos, especialmente en aquellos alejados del mundo universitario (Mattelart \& Mattelart, 1970), predomina una mirada idealizadora que se concentra en un determinado tipo de joven, descuidando la existencia de jóvenes trabajadores, empleados y campesinos.

Luego del golpe de estado de 1973, la investigación en juventud reaparece paralelamente al surgimiento de las protestas contra el gobierno de la época, en las cuales participan activamente grupos de jóvenes, especialmente populares. Precisamente es hacia estos jóvenes que se desplaza la investigación en ese período, tomando como centro el intento de explicar su participación en las protestas contra la dictadura militar. En un conjunto de investigadores prevaleció una mirada estigmatizadora de los jóvenes populares como "jóvenes problema" (i.e., delincuentes, drogadictos), mientras que otros los describieron como contestatarios, combativos y solidarios (Silva, 1999). Entre estas posturas, uno de los enfoques más citados que se utilizó para explicar el comportamiento de los jóvenes populares fue el de la anomia social, donde se entendía que los jóvenes populares desarrollaban conductas violentas (con y sin contenido político) producto de las transformaciones estructurales impulsadas por el régimen militar, entre las cuales estaba la desarticulación de las redes de seguridad y solidaridad tradicionales de los sectores populares chilenos. Esta transformación dejaba a los jóvenes en una situación de indefensión y falta de referentes sociales y normativos, ante la cual la única respuesta posible era la violencia anómica (Valenzuela, 1982).

Con la recuperación de la democracia hacia los años 90, la investigación en juventud se escinde para responder a distintas situaciones y demandas. En primer lugar, se estudian las causas de la progresiva desafección de los jóvenes por los espacios de participación política, intentando explicar el desplazamiento de este grupo social hacia posiciones consumistas, hedonistas e individualistas (Hopenhayn, 1990). En segundo lugar, se estudian los efectos de la dictadura en los jóvenes, acuñándose el término daño psicosocial para describir la situación subjetiva y los efectos del régimen militar en los jóvenes populares, así como para justificar la prioridad que debía asignarse a este grupo en las políticas públicas post dictadura (Asún, Alfaro \& Morales, 1994).

Desde otra vertiente de investigación, quizás tratando de responder a lo anterior, se intentó clarificar las principales demandas y frustraciones de los jóvenes con las instituciones sociales, con el fin de adecuar las políticas públicas a sus requerimientos. En esta línea se ha estudiado especialmente la subjetividad juvenil, sus estilos de sociabilidad, uso del tiempo libre y expectativas de vida, mostrando fuertes demandas de los jóvenes para alcanzar la integración social y su confianza en la educación como principal herramienta para lograrlo (Instituto Nacional de la Juventud [INJUV], 2006). Desde aquí se ha tendido a buscar resultados que reflejen la opinión modal de los jóvenes y a utilizar herramientas cuantitativas: encuestas, cuestionarios e índices.

Hacia fines de la década de los 90 y comienzos del siglo XXI, se desarrolla una línea de investigación basada en criterios teórico-metodológicos complementarios con la anterior vertiente: se trata de estudios cualitativos que profundizan la indagación en la subjetividad juvenil en grupos identitarios particulares (grafiteros, hip hop, miembros de barras bravas, entre otros). Esta corriente enfatiza el carácter rupturista y contestatario de estas identidades juveniles frente al discurso "integracionista" que surge de las encuestas sociales (Zarzuri $\&$ Ganter, 2005).

En suma, a pesar de la deriva temática en los estudios de juventud, es posible detectar dos constantes: en primer lugar, el estudio de lo juvenil parece ser un espacio privilegiado para la investigación de las transformaciones sociales de cada período y, en segundo lugar, la mirada de los investigadores sobre los jóvenes siempre ha girado en torno a su conflictiva integración social, ya sea por falta de espacios y oportunida- 
des o por su rechazo a la estructura social a la cual se les ofrece integrarse. La presente investigación recoge ambas tendencias, realizando una comparación entre dos períodos -lo que permite reflexionar acerca del impacto en la subjetividad juvenil que han tenido ciertas transformaciones a nivel país- y retomando los conceptos de anomia y alienación como nociones que permiten pensar el problema actual de integración/ exclusión, en el marco de una sociedad que ha sido calificada como hedonista e individualista (Lasch, 1991).

\section{Procesos de Integración Social y} Normativa: los Conceptos de Anomia y Alienación Psicosocial

Las nociones de alienación y de anomia describen negativamente los procesos de integración de los individuos al mundo social del que forman parte. Se trata de nociones que dan cuenta tanto de condiciones propias de la vida social como de la percepción subjetiva acerca de ellas. De lo anterior dan cuenta, en primer lugar, los trabajos inaugurados por la tradición marxista, en el caso del concepto de alienación, y por la perspectiva inspirada en los trabajos de Durkheim y Merton, en el caso del concepto de anomia. Por otra parte, el carácter psicosocial de ambos conceptos está dado por las dimensiones subjetivas implicadas, pues mediante estos conceptos se trata de describir, más que las condiciones "estructurales" de la vida social, el modo como los individuos experimentan dichas condiciones y las hacen parte de la percepción de sí mismos en relación a su entorno social. Por lo tanto, si bien el origen de estos conceptos se ubica en una perspectiva sociológica, socio-histórica o socio-cultural, el desarrollo de las ciencias sociales ha incorporado crecientemente la dimensión subjetiva implicada en el análisis de tales condiciones, ya sea como efectos de la misma o como parte de una unidad "dialéctica" por la cual el mundo social en sus condiciones "objetivas" encuentra en la experiencia de las personas un modo de reproducción o de reacción a ellas.

El hecho de reunir ambos conceptos en una escala resulta pertinente en función de la articulación socio-subjetiva necesaria en ambos, aun cuando describen diferentes perspectivas respecto a la percepción subjetiva del mundo social. En efecto, mediante el concepto de alienación se pone el acento en el extrañamiento que experimentan los individuos respecto de las condiciones que les ofrece (o no) la sociedad, incluyendo los aspectos materiales (e.g., resultantes del medio laboral o educativo), las condiciones propiamente psicosociales de la relación con otros y el grado de control (o de impotencia) que pueden ejercer sobre ellas. En cambio, mediante el concepto de anomia se pone el acento en un aspecto específico del mundo social, sea este "objetivo", en el sentido que define reglas o normas necesarias al ordenamiento social, o subjetivo, en el sentido de la experiencia de ser parte de dicho ordenamiento y de hacerlo propio. Dicha articulación queda reflejada en el hecho que la escala de alienación incluye como una de sus dimensiones a la anomia psicosocial.

\section{Sobre el Concepto de Alienación}

Mediante el concepto de alienación, la tradición sociológica de inspiración marxista puso de relieve la distancia o extrañamiento del individuo respecto de las condiciones sociales de las cuales es tanto su productor como su efecto. Las consecuencias de un estado de cosas que no reintegra los resultados del trabajo a sus productores inmediatos y que establece desiguales condiciones sociales para el desarrollo subjetivo pueden entenderse, ya desde el punto de vista de la organización social, económica y política, como desde la perspectiva de la experiencia que los individuos hacen de ella, experimentada en función de la relación a otros y de la relación a sí mismos, es decir, a su propia posición en el mundo social del que forman parte. Adam Schaff (1979) revisó este concepto distinguiendo dos aspectos: a) alienación (objetiva) y b) alienación de sí mismo (subjetiva). El primero alude a la relación de la persona con los resultados de su actividad de producción, en la cual estos aparecen separados de sus productores; el segundo se refiere a que esta relación se vive como separación del individuo respecto de la sociedad, de los demás hombres y de sí mismo. Por lo tanto, ambos conceptos 
designan relaciones sociales y, en ese sentido, su comprensión pasa necesariamente por un análisis sociológico. Sin embargo, sobre la base de las condiciones sociales en las cuales surge, la alienación subjetiva (o, para nuestros efectos, psicosocial) adquiere relativa especificidad en la conciencia y la percepción individual.

Para Melvin Seeman es posible establecer una serie de indicadores subjetivos del fenómeno de la alienación (impotencia, ausencia de significación, ausencia de normas, aislamiento, ausencia de autorrealización), los que estarían asociados a determinadas evoluciones de las estructuras sociales, así como a sus consecuencias en el plano psicosocial (pasividad política, repulsión al trabajo, hostilidad, desorden mental, disminución de la capacidad de aprendizaje) (Seeman, 1959, 1971). Las dimensiones subjetivas que Seeman destaca al respecto son: a) la impotencia, entendida como "la probabilidad que posee un individuo de esperar que su comportamiento tenga -o no- influencia sobre la aparición de acontecimientos particulares en su mundo social" (Seeman, 1971, p. 97); b) la ausencia de significación, refiriéndose a la incomprensión de la significación de los actos y acontecimientos en los cuales está comprometido el individuo; c) la ausencia de normas, relacionada con la noción de anomia, que se refiere a la incapacidad de poner en acción otros medios que los no aprobados para realizar sus fines; d) el aislamiento, que supone un sentimiento de soledad, abandono y, al mismo tiempo, una situación en la que se le asigna escaso valor a los objetivos y valores que son centrales en una sociedad dada; y e) el auto-extrañamiento, referido a una situación en la que el individuo está "dolorosamente consciente de la diferencia entre la ideal imagen de sí mismo y su ser real..." (Seeman, 1971, p. 98). Dwight Dean (1961) considera que, de estos indicadores o dimensiones de la alienación, es necesario resaltar tres: impotencia, aislamiento social y anomia. En base a este criterio se construye la escala de alienación descrita en la sección metodológica de este artículo y permite entender por qué la alienación aparece como la variable principal del estudio (integrando a la anomia como uno de sus componentes). La impotencia es definida como una baja expectativa de control sobre los acontecimientos sociales que afectan la vida del individuo; el aislamiento social se define como el sentimiento o percepción subjetiva de distancia y separación respecto a los demás -abandono y soledad-; y la anomia se define como la percepción subjetiva de ausencia de normas. Es en esta perspectiva teórico-metodológica que se inscribe el presente estudio sobre la experiencia de alienación juvenil, manteniendo una orientación análoga a la desarrollada por Lacourse, Villeneuve y Claes (2003).

\section{Sobre el Concepto de Anomia}

El concepto anomia fue introducido por primera vez de manera sistemática en la tradición sociológica por Emile Durkheim (Girola, 2000) ${ }^{1}$, refiriéndose con él a una situación social caracterizada por la falta de reglas. Siguiendo la definición original de Durkheim, desarrollada a partir de la idea que la división social del trabajo no produce contactos lo bastante eficaces entre sus miembros ni regulaciones adecuadas de las relaciones sociales, la expresión se refiere a una desorganización moral, en la que no hay un orden normativo compartido por la mayoría de los miembros de una sociedad, de modo que los intereses particulares no logran vincularse a un interés general.

Según Girola (2005), existiría cierto consenso en que en la obra de Durkheim el concepto de anomia tendría al menos dos sentidos distintos. El primero de ellos, aparecido en La división del trabajo social (1893/1982), correspondería a una falta de normas ocasionada por transformaciones sociales excesivamente rápidas, siendo una situación anómala transitoria agravada por el progresivo debilitamiento de la conciencia colectiva. El segundo sentido habría aparecido en El suicidio (1897/1987) y se referiría a que, en el marco de transformaciones súbitas, la sociedad dejaría de ejercer un papel regulador de las pasiones y aspiraciones de los individuos, disminuyendo su capacidad de imponer límites a lo que la

\footnotetext{
En honor a la fidelidad histórica, se debe mencionar que fue Jean Marie Guyau quien lo utilizó por primera vez en su libro Esquisse d'une morale sans obligation ni sanction (1885/1978).
} 
gente puede desear o hacer. De este modo, la anomia pasaría a ser no ya la falta de normas y reglas, sino la falta de vigencia de las normas en la vida cotidiana de la gente, otorgándole un sentido con énfasis subjetivo. Este segundo sentido del concepto es el que se rescata en la noción psicosocial de la anomia (MacIver, 1950; Riesman, Glazer \& Denney, 1964) que alude al estado mental en que el sentido de cohesión social de los individuos está debilitado, es decir, las reglas, normas y fines sociales no tienen vigencia en su vida diaria. De esto se desprende lo que posteriormente Rosner (1974) señaló como uno de los rasgos de ese estado psicológico, a saber, la imposibilidad de los individuos de prever las reacciones y conductas de sus semejantes, para determinar modelos de conducta, impedimento que se vería explicado por la falta de códigos compartidos. Siguiendo a Srole (1956), esto se vería acompañado de una percepción del entorno -y una autopercepción- como desintegrado y con una falta de involucramiento de los individuos con el medio.

Actualmente, los estudios que abordan la problemática de la anomia son relativamente escasos, tanto desde las reformulaciones de las teorías de Durkheim y Merton, como desde la perspectiva psicosocial. En el primer caso se encuentran fundamentalmente estudios de criminología, orientados a explorar casos particulares de conducta desviada o a indagar la relación de la conducta desviada con variables tales como el apoyo social, el capital social y la participación (Passas \& Agnew, 1997). En el caso de los estudios realizados desde la perspectiva psicosocial, se encuentran investigaciones que abordan la relación entre autoritarismo, anomia e identidad nacional (Blank, 2003); apoyo parental, participación religiosa y coherencia social percibida por adolescentes (Bjarnason, 1998); anomia, locus de control, autoestima, apoyo social y experiencias vitales negativas (De Man, Labreche-Gauthier \& Leduc, 1993), entre otras.

No obstante, la ruptura de marcos normativos y valorativos, con la consecuente sensación de vacuidad, aislamiento y el carácter impredecible del orden social, ha sido tematizada de diversas maneras, sin abordarla necesariamente desde el concepto de anomia o alienación. En esta línea encontramos a Lasch (1991), quien con el nombre la cultura del narcisismo denomina una era en la que han declinado instituciones tradicionales tales como el Estado y la familia y en que impera el individualismo y la incertidumbre respecto del futuro. Por su parte, Lipovetsky (1986) retrata a una sociedad europea centrada en la realización personal y el disfrute, marcada por un individualismo extremo, alimentado por la cultura neoliberal. La ética resultante podría ser denominada, siguiendo a Guyau, una moral sin sanción ni obligación (Girola, 2005).

Finalmente, Beck (1998), aunque desde una perspectiva distinta, aporta a esta reflexión sobre la crisis de cohesión social en las sociedades contemporáneas, particularmente la alemana. Según este autor, con posterioridad a la segunda guerra mundial se habría producido un aumento generalizado de la calidad de vida en dicho país y sobre la base de un estándar de vida relativamente alto y la existencia de una seguridad social también elevada, se habría quebrado con la continuidad histórica de las amarras tradicionales de las personas a condiciones de clase y a la familia. En otras palabras, con la desaparición de ambientes y culturas típicamente obreras y con la fragmentación de la familia, las personas se ven obligadas a depender de sí mismas y de su propia suerte en el mercado laboral y, en general, en la definición de su proyecto vital. Esta individualización se ve acompañada de un aumento de posibilidades y libertades, pero también de riesgos, angustias y sentimientos de abandono y soledad. La incertidumbre generada por este riesgo permanente es paliada, según Beck, con una institucionalización y estandarización de las formas de vida, a través de la cultura del consumo: los individuos desamarrados (liberados) se hacen dependientes del mercado. Las formas de existencia resultantes son el consumo masivo de viviendas, amoblados y artículos del consumo diario. De este modo, se podría decir que se produce una "ritualización" de la vida cotidiana: importa la forma, no el para qué.

Esta línea de pensamiento ha sido desarrollada en Chile por Norbert Lechner, quien en el año 2000 proponía un desajuste entre el desarrollo macroeconómico y la 
sensación de seguridad de los ciudadanos, producido por un proceso de modernización acelerado, en el que el cambio de los sistemas funcionales no fue acompañado de una reflexión acerca de nuestra sociedad y de un "nosotros" (Lechner, 2000). En términos durkhemianos, estaríamos ante un proceso acelerado y abrupto de cambio que mina los códigos compartidos, perdiendo los individuos el enraizamiento al tejido social que permite generar vínculos y lazos de sociabilidad. En este contexto, el proceso de individualización adquiere las características de un individualismo negativo o asocial (Programa de las Naciones Unidas para el Desarrollo [PNUD], 2002). El desamarre de instituciones tradicionales y la flexibilización de las formas de vida se ven acompañados de una organización de los sistemas sociales que no provee los recursos para la autodeterminación. Esto hace que la vida social se experimente como agobio y amenaza, como naturalización del mundo social, centrando su sociabilidad en micro espacios, como la familia o los amigos más cercanos.

El presente estudio se inscribe en la tradición reflexiva descrita, en tanto interesa considerar la anomia y la alienación como fenómenos que dan cuenta -negativa, crítica o conflictivamente- de la experiencia de integración/desintegración de los jóvenes respecto al mundo social en el que existen y se desenvuelven como personas. A partir de estos dos conceptos interesa abordar tanto la condición normativa (nomos) que plantea el mundo social, en sus aspectos objetivos e institucionalizados (reglas claras de participación o de exclusión social, oportunidades de acceso a beneficios sociales) y en sus aspectos subjetivos (percepción de estabilidad del mundo social, de normas que regulan interacciones), como la relación del individuo respecto a "los otros" (proximidad, extrañamiento, confianza/desconfianza), nuevamente en su condición objetiva y subjetiva o psicosocial.

\section{Método}

\section{Muestra y Procedimiento}

Considerando que el propósito de esta investigación es comparar los niveles de anomia y alienación de los jóvenes san- tiaguinos entre 1989 y 2007, se replicaron tanto los instrumentos aplicados como el procedimiento de muestreo utilizado en la primera oportunidad.

Además, ya que solo se disponía del informe de resultados de la investigación de 1989 y no de la base de datos, se replicaron también los procedimientos de construcción de escalas y análisis estadísticos usados. Esto constituye una limitación del presente estudio, pues no se pudo aplicar técnicas de análisis habituales actualmente, pero permite la comparación de resultados entre ambas investigaciones.

Para el procedimiento de selección de la muestra del año 1989 se construyó una tipología de colegios de la Región Metropolitana en función del nivel socioeconómico esperado de sus estudiantes, clasificando cada colegio en nivel social bajo, medio o alto. Los criterios utilizados para ello fueron: a) la dependencia administrativa (distinguiendo colegios privados, particulares subvencionados y municipales) y b) la comuna de ubicación (las que fueron clasificadas en comunas mayoritariamente habitadas por personas de nivel social alto, medio y bajo, según clasificaciones de empresas de estudios de mercado). Se entendió que los colegios que probablemente disponían de más estudiantes de nivel social alto eran aquellos ubicados en comunas de estrato alto y dependencia particular. Por su parte, los estudiantes de nivel social medio debían ser más frecuentes en los colegios municipales o particulares subvencionados, ubicados en comunas de estrato medio, y los estudiantes de nivel social bajo debían encontrarse principalmente en comunas de estrato bajo y colegios de dependencia municipal o particular subvencionado.

En función de los criterios anteriores se eligieron intencionadamente siete establecimientos educacionales (dos de nivel alto, dos de nivel medio y tres de nivel bajo) que cumplían con las condiciones señaladas. En el año 2007 se replicó la muestra obtenida en 1989, recurriéndose a los mismos colegios para realizar la investigación. Sin embargo, se hizo necesario reemplazar dos colegios: uno de ellos ya no existía y el otro había sufrido un desplazamiento ascendente en el nivel social de sus estudiantes, pues 
su dependencia había pasado de particular subvencionada a particular privada. Para reemplazar dichos colegios se eligieron otros lo más similares posibles a los originales, seleccionándose establecimientos de la misma comuna, sexo de sus estudiantes y dependencia. Finalmente, en el año 2007 se obtuvo una muestra muy similar a la de 1989 , como se puede corroborar en la Tabla 1.

A pesar de los esfuerzos realizados para obtener muestras idénticas, se puede observar que en el año 2007 se obtuvo alrededor de un $7 \%$ más de estudiantes mujeres y de nivel social alto. Con el fin de que las comparaciones fuesen lo más equivalentes posibles, este sesgo fue controlado ponderando los grupos, disminuyendo la importancia relativa de los segmentos sobredimensionados. Este ponderador se utilizó en todos los resultados que se presentan más adelante, pero como medida de control se comprobó que no existieran diferencias significativas en los resultados obtenidos utilizando o no el ponderador.

En ambos años, en cada uno de los establecimientos se obtuvo la autorización del Director, seleccionando al azar un curso de cuarto año medio, en el cual fue aplicado el cuestionario. La aplicación se realizó en forma auto-administrada, con un encuestador que entregaba las instrucciones y respondía dudas. Se explicó a los estudiantes que el cuestionario era parte de una investigación universitaria cuyo objetivo era conocer la forma en que los jóvenes perciben la sociedad chilena. Asimismo, se les aclaró que responder era voluntario, que nadie cono- cería las respuestas individuales y que no tendría consecuencias responder en blanco. Solo cuatro estudiantes entregaron el cuestionario completamente vacío. La aplicación tomó alrededor de 45 minutos.

\section{Instrumento}

Se aplicó prácticamente el mismo cuestionario en ambos años. El cuestionario de 1989 constaba de tres tipos de preguntas: a) preguntas de identificación del estudiante (en que se pesquisaba el sexo, la edad y el nivel social de la familia), b) preguntas que medían variables hipotéticamente asociadas a la integración social y normativa de los estudiantes (donde se encontraban la medición de la estabilidad laboral del padre, su nivel de religiosidad, el nivel de apoyo social percibido y el locus de control interno/externo del individuo) y c) escalas de anomia y alienación.

En el año 2007 solo se cambiaron tres variables: se eliminó la escala de locus de control y se modificó la forma de preguntar respecto de la estabilidad laboral y la religiosidad, con el fin de mejorar su comprensión. Las escalas que medían la integración social y normativa (escalas de anomia y alienación) se aplicaron exactamente igual que en el año 1989.

Ambas escalas están formadas por afirmaciones tipo Likert, con tres alternativas de respuesta (de acuerdo, no sé y en desacuerdo). La alternativa de acuerdo se puntuó como 1, no sé como 2 y la alternativa en desacuerdo, como 3 , valores que

Tabla 1

Muestras Obtenidas en los Años 1989 y 2007

\begin{tabular}{lcc}
\hline & Muestra año 1989 & Muestra año 2007 \\
\hline Tamaño de muestra & 363 & 343 \\
Número de colegios & 7 & 7 \\
Porcentaje de hombres & 52,6 & 45,8 \\
Porcentaje de estudiantes de nivel alto & 21,8 & 29,8 \\
$\begin{array}{l}\text { Porcentaje de estudiantes de nivel } \\
\text { medio }\end{array}$ & 51,9 & 49,4 \\
Porcentaje de estudiantes de nivel bajo & 26,3 & 20,8 \\
\hline
\end{tabular}


posteriormente se re-codificaron, de manera que obtuviera 3 puntos la respuesta que implicase más alienación o anomia y 1 la respuesta contraria, dejando siempre el 2 para la alternativa no sé. Estas puntuaciones suponen que la alternativa no sé ocupa un lugar intermedio en un continuo, lo que podría estar equivocado, pero este fue el procedimiento usado en el año 1989, por lo que se decidió replicarlo. Con el fin de cautelar que se pudiera cometer un error importante al suponer ese continuo, alternativamente se recodificó la alternativa no se de otras dos formas: a) como si se tratara de una respuesta anómica o alienada; b) como si se tratara de una respuesta no anómica o alienada (puntuándola en ambos casos con valor 1 ó 3 , dependiendo de la dirección del ítem). La correlación lineal de Pearson entre estas formas alternativas de puntuación y la puntuación original fue en ambos casos superior a 0,89 , por lo que el error no sería de gran magnitud.

La escala de anomia utilizada es de Aceituno y Drago (Aceituno, 1989), basada en las dimensiones contenidas en la Escala de Anomia de Srole (1956), y consta de 20 afirmaciones divididas en cuatro dimensiones: exclusión, que indica la percepción de indiferencia de la comunidad respecto a las necesidades individuales (5 afirmaciones), incertidumbre, referida a la percepción que el orden social se encuentra desorganizado y es impredecible (5 afirmaciones), degradación, que alude a un sentimiento de retroceso frente a objetivos anteriormente propuestos y realizables (5 afirmaciones), y extrañamiento, que es la percepción del entorno social como agresivo y hostil (5 afirmaciones) ${ }^{2}$.

La escala de alienación utilizada también es de Aceituno y Drago (Aceituno, 1989) y consta de 35 afirmaciones divididas en tres dimensiones: aislamiento social, que alude al sentimiento de distancia y separación respecto a los demás (9 afirmaciones), impotencia, que se refiere a una baja expectativa de control personal sobre los acontecimientos sociales que afectan la vida del individuo (6 afirmaciones), y anomia, que es la percepción subjetiva de ausencia de normas (20 afirmaciones). Se debe hacer notar que la dimensión anomia es medida por la escala de anomia reseñada en el párrafo anterior. De esta forma, operacionalmente la alienación incluye a la anomia, como se observa en la Figura 1.

2 Acogiendo el criterio de Valenzuela (1984), Aceituno y Drago eliminaron la dimensión de sin sentido contenida en la escala original de Srole, pues esta sería más bien una consecuencia de la percepción anómica.

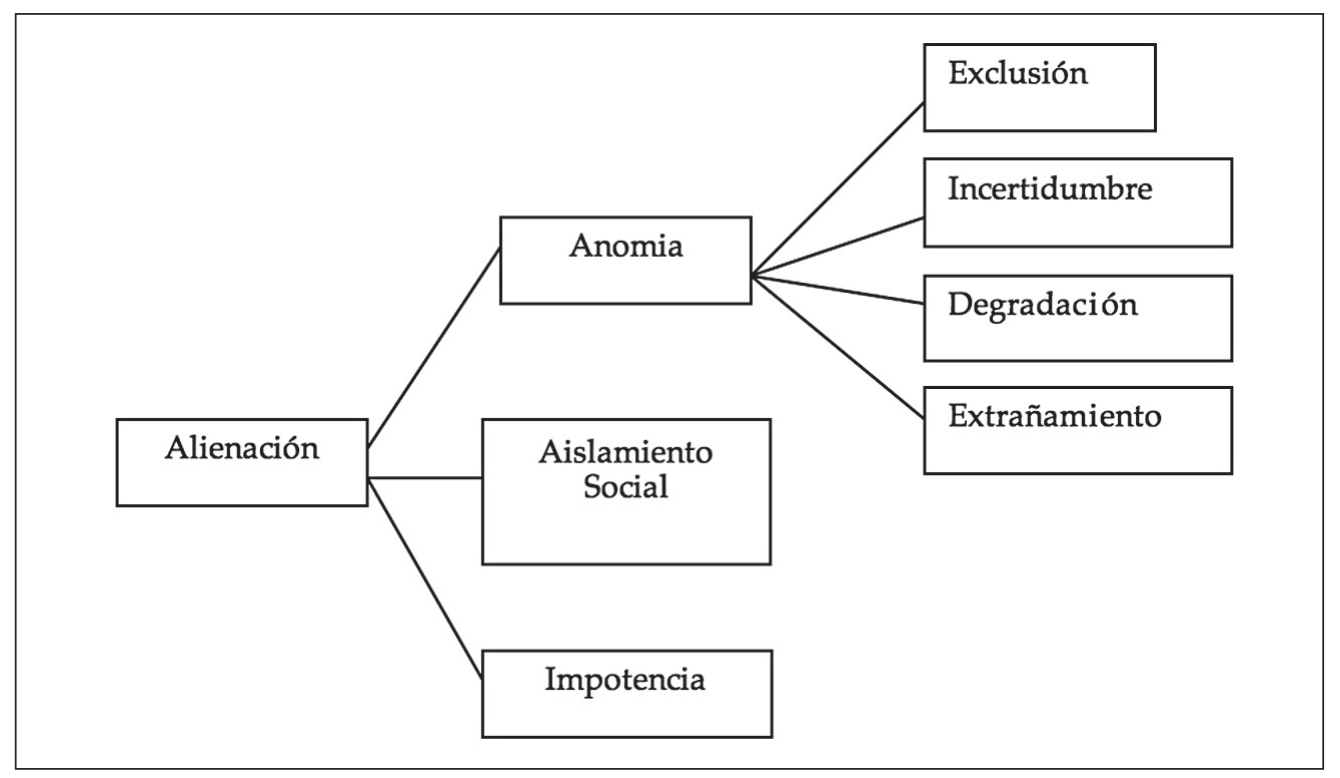

Figura 1. Modelo operacional para la medición de anomia y alienación. 


\section{Análisis de los Datos}

Respecto a la construcción de las escalas, en el año 1989 se optó por realizar los análisis psicométricos separadamente para cada sub-escala de la alienación, procedimiento que se replicó en 2007. También se repitió el análisis estadístico utilizado, que fue calcular la correlación media entre los ítems y la consistencia interna del instrumento por medio del coeficiente de dos mitades de Guttman, corregido por el procedimiento de Spearman-Brown.

En relación con los niveles de anomia y alienación en 1989 y 2007, se compararon las puntuaciones medias obtenidas en ambos años, determinando la significación y tamaño del efecto de las diferencias. Para la significación se utilizó la prueba $t$ de Student y para los tamaños del efecto el estadístico $d$ de Cohen.

\section{Resultados}

\section{Funcionamiento y Construcción de las Subescalas de Alienación}

Respecto de la subescala de anomia, la correlación media entre los 20 ítems del instrumento original fue 0,08 . Por su parte, la consistencia interna obtenida fue 0,68 . Considerando ambos valores insatisfactorios, se eliminaron seis ítems del instrumento, con lo que se logró aumentar la correlación entre los ítems a 0,14 y la consistencia interna a 0,73 . Ambos valores se encuentran en rangos aceptables. Replicando estos procedimientos, en el año 2007 se obtuvo una consistencia interna inicial de la escala de 20 ítems de 0,66 y una correlación entre los ítems de 0,08. Eliminando los mismos seis ítems que en el año 1989, se logró una consistencia interna de 0,63 y una correlación entre ítems de 0,11 . De estos resultados se concluye que los ítems que funcionaron psicométricamente mal en el año 1989 fueron los mismos en el año 2007 y que la escala de anomia alcanzó niveles de confiabilidad regulares en ambas oportunidades.

La subescala de aislamiento social de nueve ítems tuvo en el año 1989 una correlación entre ítems de 0,09 y una consistencia interna por dos mitades de 0,47 . Ya que estos resultados no eran satisfactorios, se eliminaron dos ítems, con lo que la correlación entre ítems subió a 0,12 y la consistencia interna a 0,54. En el año 2007 la sub-escala de aislamiento social tuvo inicialmente una correlación entre ítems de 0,11 y una consistencia interna de 0,48. Eliminando los mismos dos ítems que en el año 1989, la consistencia interna subió a 0,51 y la correlación entre los ítems se mantuvo en 0,11 . Se puede notar que ambos valores son análogos a los del año 1989, lo que muestra el similar funcionamiento del instrumento. Como también es evidente, el nivel de consistencia interna de esta subescala fue poco satisfactorio en ambas oportunidades.

Finalmente, con relación a la subescala de impotencia de seis ítems, en el año 1989 tuvo una correlación entre ítems de 0,18 y una consistencia interna por dos mitades de 0,66 . A diferencia de los instrumentos anteriores, ninguna eliminación de afirmaciones permitía subir estos índices, por lo que se decidió mantener la escala con la totalidad de las afirmaciones originales. En el año 2007 la correlación entre los ítems fue 0,16 y la consistencia interna, 0,53. Tampoco este año fue posible eliminar ninguna afirmación para aumentar estos indicadores, por lo que se optó igualmente por mantener el instrumento original completo. Se puede notar que con esta subescala tampoco se obtuvieron niveles de consistencia interna satisfactorios.

En suma, todas las escalas funcionaron de manera muy similar en ambas aplicaciones, funcionamiento que no alcanzó niveles óptimos en ningún instrumento, siendo el de anomia el de mejor consistencia interna. No obstante lo anterior, el alfa de Cronbach del instrumento conjunto (puntuación de alienación) fue aceptable, considerando que se trata de una escala con tres subescalas en su interior: 0,70.

Dada la solo regular confiabilidad de los instrumentos, se decidió utilizar 0,01 como nivel de significación para las pruebas de hipótesis, de manera que, en un contexto de mediciones menos precisas, se deba encontrar diferencias mayores para concluir la existencia de diferencias significativas entre los grupos o las mediciones que se comparen. 


\section{Niveles Comparados de Anomia y Alienación}

Con relación a los niveles de alienación encontrados en 1989 respecto a los de 2007, se puede observar en la Tabla 2 que la puntuación media obtenida por los participantes fue moderadamente menor en 2007 que en 1989 (la media disminuyó 1,1 puntos). La diferencia de las medias entre ambas mediciones es significativa, $t(653)=5,27, p$ $=0,000$, pero el tamaño del efecto de esta diferencia es medio-bajo $(d=0,41)$.

Observando en la Figura 2 la distribución de las puntuaciones obtenidas por los estudiantes en la escala de alienación, se puede notar que, a excepción de una tendencia a una mayor proporción de puntuaciones medio-bajas en el año 2007, ambas distribuciones son ampliamente similares.

Respecto a los resultados de la subescala de anomia, en la Tabla 3 se observa también una disminución de 1,1 puntos en la media y cierta reducción de la heterogeneidad de la distribución. La diferencia de las medias de ambas mediciones también es significativa, $t(653)=3,24, p=0,001$, pero en este caso el tamaño del efecto de esta diferencia es bajo $(d=0,25)$.

En cuanto a la distribución de las puntuaciones obtenidas por los estudiantes en la subescala de anomia, se puede notar en la Figura 3 que, al igual que en el caso de la alienación, ambas distribuciones son ampliamente similares, a excepción de una leve disminución de las puntuaciones medio-altas.

En tanto, para la subescala de impo-

Tabla 2

Estadísticos en Alienación en 1989 y 2007

\begin{tabular}{lrc}
\hline Estadístico & 1989 & 2007 \\
\hline Intervalo de valores de la escala & $10-30$ & $10-30$ \\
Promedio & 18,73 & 17,61 \\
Desviación estándar & 2,89 & 2,47 \\
\hline
\end{tabular}

Nota . Alienación $=($ Impotencia $/ 6+$ Aislamiento social $/ 7+$ Anomia $/$ 14) / 3) * 10 .

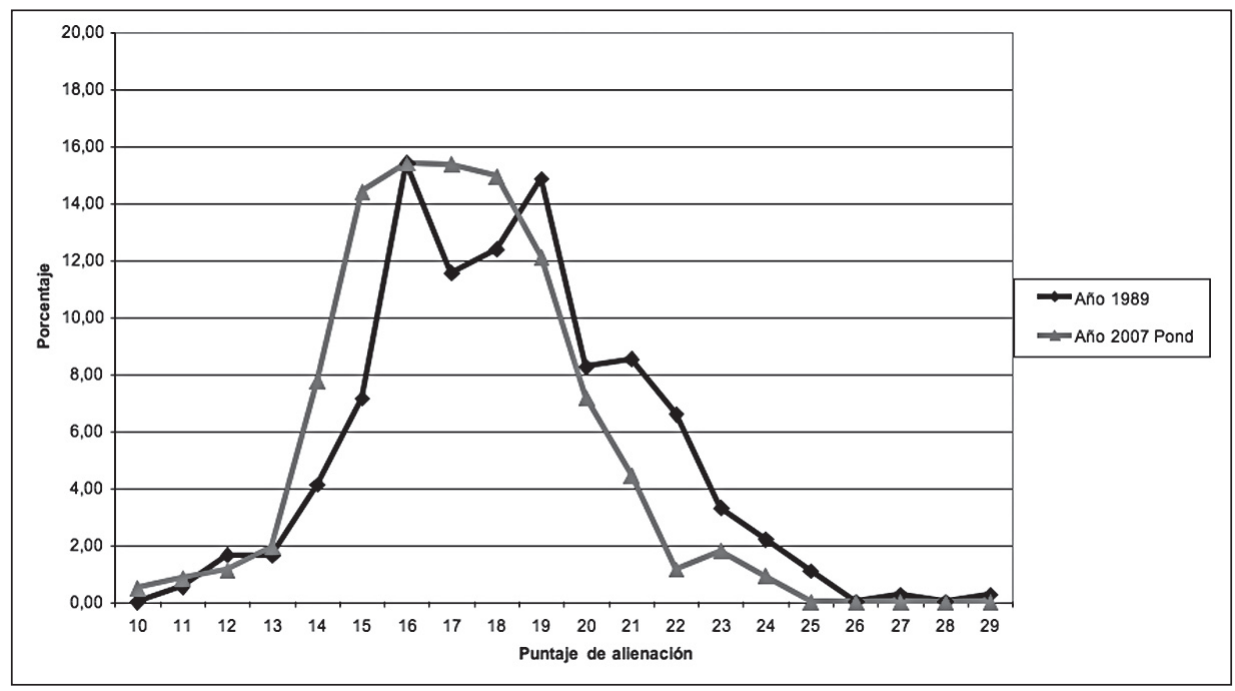

Figura 2. Distribución de las puntuaciones ponderadas de alienación. 
Tabla 3

Estadísticos en Anomia en 1989 y 2007

\begin{tabular}{lcc}
\hline Estadístico & 1989 & 2007 \\
\hline Intervalo de valores de la escala & $14-42$ & $14-42$ \\
Promedio & 25,92 & 24,78 \\
Desviación estándar & 4,66 & 4,36 \\
\hline
\end{tabular}

Nota . Anomia = sumatoria de la puntuación obtenida por los estudiantes en los 14 ítems de la subescala.

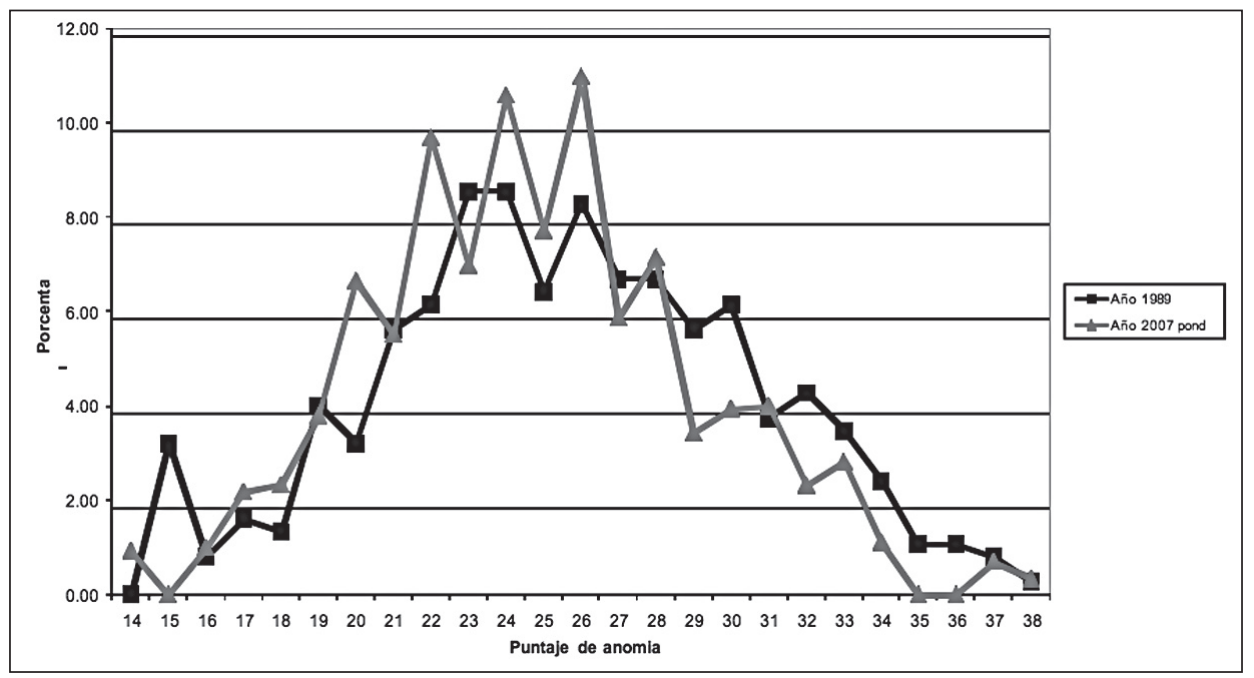

Figura 3. Distribución de las puntuaciones ponderadas de anomia.

tencia en la Tabla 4 se muestra el mismo patrón de comportamiento que las escalas anteriores: una disminución de 1,2 puntos en el promedio, diferencia que es estadísticamente significativa, $t(671)=6,85, p=$ 0,000 , y que constituye un efecto de magnitud intermedio $(d=0,53)$.

Finalmente, respecto a la subescala de aislamiento social, se observa en la Tabla 5 una situación diferente. La diferencia de puntuaciones promedio entre los años 1989 y 2007 es solo de 0,45 puntos, la que no es significativa, $t(668)=2,12, p=0,034$, y tiene un tamaño del efecto mínimo $(d=0,16)$.

En suma, para casi todas las escalas y subescalas se detectaron diferencias signifi-

Tabla 4

Estadísticos en Impotencia en 1989 y 2007

\begin{tabular}{lll}
\hline Estadístico & 1989 & 2007 \\
\hline Intervalo de valores de la escala & $6-18$ & $6-18$ \\
Promedio & 9,26 & 8,00 \\
Desviación estándar & 2,59 & 2,07 \\
\hline
\end{tabular}

Nota. Impotencia = sumatoria de la puntuación obtenida por los estudiantes en los 6 ítems de la subescala. 
Tabla 5

Estadísticos en Aislamiento Social en 1989 y 2007

\begin{tabular}{lrr}
\hline Estadístico & 1989 & \multicolumn{1}{c}{2007} \\
\hline Intervalo de valores de la escala & $7-21$ & $7-21$ \\
Promedio & 15,58 & 15,13 \\
Desviación estándar & 2,82 & 2,61 \\
\hline
\end{tabular}

Nota . Aislamiento Social = sumatoria de la puntuación obtenida por los estudiantes en los 7 ítems de la subescala.

cativas en las puntuaciones promedio entre los años 1989 y 2007, pero esas diferencias fueron moderadas o bajas y no afectaron mayormente la forma de distribución de las variables. En el caso de la subescala de aislamiento social, la magnitud de la diferencia no permite descartar que se deba al azar. La excepción fue la puntuación en la subescala de impotencia, en la que se encontró una disminución de magnitud más relevante entre los años 1989 y 2007.

\section{Discusión y Conclusiones}

En 1989 Chile se encontraba en las postrimerías de un período dictatorial que limitó fuertemente las libertades políticas y de expresión ciudadana y desintegró muchas de las formas tradicionales de sociabilidad entre los chilenos, especialmente dentro de los sectores populares. La pérdida de importancia de los sindicatos y las juntas vecinales, el deterioro de los sistemas de protección social, la privatización y aumento de las desigualdades al interior del sistema educativo, son algunos ejemplos de ello. Paralelamente, pese a que en el último trienio del gobierno militar se experimentó un proceso de mejoramiento de las condiciones económicas, una altísima proporción de la población vivía en condiciones de pobreza y la desigualdad en el país había aumentado claramente respecto del nivel al inicio de los años 70 (Larrañaga, 2001).

En cambio, en el año 2007 Chile presentaba condiciones bastante diferentes en casi todos estos indicadores. Existe un amplio sistema de libertades políticas y de expresión, se respetan las libertades de agrupación y negociación, ha habido un me- joramiento de los sistemas de protección social, han aumentado los salarios reales por hora y la inversión en educación, lo que ha facilitado que los niveles de cobertura de los sistemas medio y superior se incrementen sustantivamente (INJUV, 2006). Desde el punto de vista del acceso al consumo, los cambios son aún más nítidos: los chilenos tienen más acceso a tecnología, vivienda y bienes de consumo que en el año 1989. La economía del país ha crecido a un promedio cercano al 5,5\% durante todo el período, lo que, sumado al desarrollo de los sistemas de protección social, ha permitido disminuir los niveles de pobreza e indigencia de los chilenos (INJUV, 2006). Parece difícil negar, en el marco de esta mirada global, que los jóvenes de hoy se enfrentan a un mundo que ofrece mejores expectativas que el de hace 20 años.

No obstante, los resultados de esta investigación muestran que los niveles de integración social y normativa de los jóvenes del año 2007 no difieren demasiado de lo registrado en el año 1989. Sintetizando, se puede decir que los jóvenes del año 2007 efectivamente manifiestan niveles más bajos de alienación frente a la sociedad chilena, pero esta disminución es nula en el caso de la percepción de aislamiento social, baja con relación a la anomia y de magnitud destacable solo en el caso de la percepción de impotencia.

¿Cómo podemos interpretar la paradoja de vivir en una sociedad y un país que ofrece y permite mejores condiciones materiales de vida, con jóvenes que poseen similares percepciones de desintegración normativa que hace 20 años?

Una primera interpretación podría con- 
sistir en suponer que el nivel de integración social y normativa de los jóvenes constituye, en el marco de una sociedad capitalista con predominio de roles adquiridos, una constante fundamentalmente relacionada con el desarrollo biológico y evolutivo de estos. Aunque esta interpretación es plausible, no resulta particularmente convincente, pues tiende a simplificar la dimensión subjetiva de la vida social y a reificar la condición juvenil.

Una segunda vía de interpretación, de carácter más socio-estructural, implica preguntarse por aquellos aspectos propios del Chile de 1989 que no hayan experimentado cambios sustantivos respecto a la situación predominante en 2007 y que podrían estar explicando la mantención de los niveles de anomia y alienación. Diversas investigaciones señalan que la situación chilena no ha cambiado demasiado entre estos años en dos aspectos: en el nivel de desigualdad social y el nivel de reproducción generacional de las diferencias sociales. Así, Chile tenía a nivel nacional en el año 1990 un índice Gini de 0,58, mientras que dicho indicador alcanzaba un 0,57 en el año 2003 (INJUV, 2006). Sobre la movilidad intergeneracional, se constata que Chile exhibe un bajo grado de movilidad social, lo cual indica una particularmente desigual distribución de las oportunidades, a pesar de un leve mejoramiento de este indicador a partir de 1990 (Núñez \& Risco, 2004).

Ambas condiciones de estabilidad podrían explicar los persistentes niveles de anomia y alienación de los jóvenes. Lo anterior se refuerza si se considera que los dos problemas principales de Chile como sociedad democrática son, para los jóvenes, la desigualdad y la falta de oportunidades (INJUV, 2006).

También esto explicaría por qué la única dimensión en que se encontró diferencias sustantivas fuera la impotencia, ya que posiblemente la sensación de incapacidad frente al futuro es más sensible a los cambios en la magnitud absoluta de posibilidades materiales disponibles que la percepción de aislamiento social o desestructuración normativa.

En suma, los resultados de esta investigación parecen indicar que los jóvenes son más sensibles a la desigualdad y a una estructura de oportunidades percibida como injusta, que a sus condiciones materiales de vida, cuando evalúan su satisfacción con la sociedad en que viven. Esta sensibilidad podría estar relacionada con el momento en el que se encuentran: concluyendo la educación secundaria y, por ende, con las miradas puestas en los próximos pasos educacionales y/o laborales. Este momento vital es crucial, en tanto se hacen patentes la desigualdad y diferencias sociales en las posibilidades de elección y decisiones que configuran el proyecto personal.

Esta segunda hipótesis resulta más satisfactoria, pero deja sin explicar que el menor cambio se dé en la subescala de aislamiento social. Para explicar ese fenómeno, se propone una tercera hipótesis complementaria de la segunda: la evolución de Chile en estos 20 últimos años no ha brindado redes de solidaridad o seguridad que permitan a muchos jóvenes sentirse amparados por su pertenencia a nuestra sociedad. A este respecto se debe recordar que diversos Informes de Desarrollo Humano en Chile han expresado que el desarrollo económico chileno convive con una fuerte carencia en las estrategias de sociabilidad y solidaridad e incluso ha tendido a desdibujar nuestra percepción como colectivo social (PNUD, 2000), todo esto en un contexto de crecientes niveles de individualización general y juvenil (INJUV-PNUD, 2004).

Desde esta perspectiva, y siguiendo algunas ideas de Lechner (2000), la estabilidad en la anomia y alienación de los jóvenes podría ser consecuencia de las dificultades que se tiene en nuestro país para construir proyectos colectivos y sentido de pertenencia. Particularmente, en las generaciones jóvenes esta falta de identificación con un proyecto colectivo puede hacer que, ante un cambio vital en que predomina la incertidumbre -como es la salida de la enseñanza media-, el aislamiento y la soledad se sientan con mayor fuerza.

Lamentablemente, no se dispone de información empírica que permita optar o evaluar estas tres hipótesis. Solo la realización de investigaciones comparativas a nivel internacional en países que tengan distintos niveles de condiciones materiales 
de vida y redes de solidaridad o sentido colectivo, podría entregar mayores luces.

Se propone, entonces, avanzar en la dirección de la realización de esos estudios, lo que podría mejorar sustantivamente el conocimiento de las condiciones que inciden en la percepción de integración social de un actor clave de las sociedades latinoamericanas, como son los jóvenes.

\section{Referencias}

Aceituno, R. (1989). Anomia y alienación en estudiantes de cuarto medio de Santiago. Un estudio exploratorio. Memoria no publicada para optar al título de Psicólogo, Departamento de Psicología, Facultad de Ciencias Sociales, Universidad de Chile, Santiago, Chile.

Agurto, I., Canales, M. \& De la Maza, G. (1985). Juventud chilena: razones y subversiones. Santiago: ECO-FOLICO-SEPADE.

Asún, D., Alfaro, J. \& Morales, G. (1994). Análisis crítico del uso de categorías y estrategias para el estudio e intervención psicosocial con jóvenes en Chile. Revista Chilena de Psicología, 15(1), 5-14.

Beck, U. (1998). La sociedad del riesgo. Hacia una nueva modernidad. Barcelona: Paidós.

Bjarnason, T. (1998). Parents, religion and perceived social coherence: A Durkheimian framework of adolescent anomie. Journal for the Scientific Study of Religion, 37, 742-754.

Blank, T. (2003). Determinants of national identity in East and West Germany: An empirical comparison of theories on the significance of authoritarianism, anomie, and general selfesteem. Political Psychology, 24, 259-288.

Dean, D. G. (1961). Alienation: Its meaning and measurement. American Sociological Review, 26, 753-758.

De Man, A. F., Labreche-Gauthier, L. \& Leduc, C. P. (1993). Correlates of anomie in French-Canadian adolescents. The Journal of Social Psychology, $133,141-145$.

Durkheim, E. (1893/1982). La división del trabajo social. Madrid: Akal.

Durkheim, E. (1897/1987). El suicidio. Buenos Aires: Schapire.

Girola, L. (2000). ¿Cuál modernidad? Anomia en las sociedades latinoamericanas. Revista Venezolana de Economía y Ciencias Sociales, 6(2), 91-103.

Girola, L. (2005). Anomia e individualismo: del diagnóstico de la modernidad de Durkheim al pensamiento contemporáneo. Barcelona: Anthropos/ Universidad Autónoma Metropolitana.

Guyau, J. M. (1885/1978). Esbozo de una moral sin obligación ni sanción. Madrid: Júcar.

Hopenhayn, M. (1990). Un día después de la muerte de una revolución. Revista Estudios Públicos, 37, 34-43.
Instituto Nacional de la Juventud (2006). Segundo Informe Nacional de Juventud. Santiago, Chile: Autor.

Instituto Nacional de la Juventud-Programa de las Naciones Unidas para el Desarrollo (2004). Transformaciones culturales e identidad juvenil en Chile. Santiago, Chile: Autores.

Lacourse, E., Villeneuve, M. \& Claes, M. (2003). Theoretical structure of adolescent alienation: A multigroup confirmatory factor analysis. Adolescence, 38, 639-650.

Larrañaga, O. (2001). Distribución de ingresos en Chile: 1958-2001 (Documento de Trabajo). Santiago, Chile: Departamento de Economía, Universidad de Chile.

Lasch, C. (1991). La cultura del narcisismo. Barcelona: Andrés Bello.

Lechner, N. (2000). Desafíos de un desarrollo humano: individualización y capital social. En B. Kliksberg $\&$ L. Tomassini (Comps.), Capital social y cultura: claves estratégicas para el desarrollo (pp. 101-127). Buenos Aires: Fondo de Cultura Económica.

Lipovetsky, G. (1986). La era del vacío. Ensayo sobre el individualismo contemporáneo. Barcelona: Anagrama.

MacIver, R. (1950). The rampants we ward. New York: MacMillan.

Mattelart, A. \& Mattelart, M. (1970). Juventud chilena: rebeldía y conformismo. Santiago, Chile: Universitaria.

Núñez, J. \& Risco, C. (2004). Movilidad intergeneracional del ingreso en un país en desarrollo: el caso de Chile (Documento de Trabajo). Santiago, Chile: Universidad de Chile, Departamento de Economía.

Passas, N. \& Agnew, R. (1997). The future of anomie theory. Boston: Northeastern University Press.

Programa de las Naciones Unidas para el Desarrollo (2000). Desarrollo humano en Chile 2000. Más sociedad para gobernar el futuro. Santiago, Chile: Autor.

Programa de las Naciones Unidas para el Desarrollo (2002). Informe de desarrollo humano en Chile. Nosotros los chilenos: un desafio cultural. Santiago, Chile: Autor.

Riesman, D., Glazer, N. \& Denney, R. (1964). La muchedumbre solitaria. Buenos Aires: Paidós.

Rosner, M. (1974). Aliénation, fétichisme, anomie. En B. Fraenkel, Freudo-marxisme et sociologie de l'aliénation (pp. 74-125). Paris: Anthropos.

Schaff, A. (1979). La alienación como fenómeno social. Barcelona: Crítica/Grijalbo.

Seeman, M. (1959). On the meaning of alienation. American Sociological Review, 24, 783-791.

Seeman, M. (1971). Alienación: un mapa de sus territorios principales (traducido por R. Zúniga en Cuadernos de Psicología Social). Valparaíso, Chile: Ediciones Universitarias de Valparaíso.

Silva, J. (1999). Ni héroes ni malvados, solo jóvenes. Claves para iluminar la conversación sobre los jóvenes de los noventa [Versión electrónica]. Última Década, 11. Extraído el 17 Junio, 2008, de http://www.cidpa.cl/txt/11ARTI2.DOC 
Srole, L. (1956). Social integration and certain corollaries: An exploratory study. American Sociological Review, 21, 709-716.

Valenzuela, E. (1982). Los jóvenes de los ochenta. Una interpretación sociológica de la actual generación estudiantil de clase media. Santiago, Chile: SUR. Valenzuela, E. (1984). La rebelión de los jóvenes. Un estudio sobre la anomia social. Santiago, Chile: SUR.

Zarzuri, R. \& Ganter, R. (2005). Jóvenes, la diferencia como consigna: ensayos sobre la diversidad cultural juvenil. Santiago, Chile: Centro de Estudios en Seguridad Ciudadana.

Fecha de recepción: Agosto de 2008.

Fecha de aceptación: Mayo de 2009. 